\title{
Ca-NIR: A Ratiometric Near-Infrared Calcium Probe Based on Dihydroxanthene-hemicyanine Fluorophore
}

\author{
Mayeul Collot, ${ }^{* a}$ Flavien Ponsot, ${ }^{a}$ Andrey S. Klymchenko ${ }^{a}$
}

Fluorescent calcium probes are essential tools for studying the fluctuation of calcium ions in cells. Herein, we developed Ca-NIR, the first ratiometric calcium probe emitting in the near infrared region. This probe arose from the fusion of a BAPTA chelator and a dihydroxanthene-hemicyanine fluorophore. It is efficiently excited with common 630-640 nm lasers and diplays two distinct emission bands depending on the calcium concentration ( $\mathrm{Kd}=\sim 8 \mu \mathrm{M})$. The physicochemical and spectroscopic properties of Ca-NIR allowed for ratiometric imaging of calcium distribution in live cells.

Calcium ions $\left(\mathrm{Ca}^{2+}\right)$, and their movement within the cells, play an important role in many biological processes as calcium is an ubiquitous second messenger involved in numerous intracellular signalling cascades. ${ }^{1}$ For this reason, monitoring the spatiotemporal fluctuations of intracellular calcium concentration $\left[\mathrm{Ca}^{2+}\right]$ is of great importance. The tremendous expansion of fluorescence imaging techniques led to the development of various fluorescent probes including calcium probes. ${ }^{2}{ }^{3}$ Although the first fluorescent calcium probes were developed in the eighties by Tsien and colleagues, ${ }^{4}$ continuous progresses have been made in the field by improving several parameters responding to certain demand. The intensive use of the green-yellow region of the spectrum by Fluorescent Proteins (GFP and YFP) led to the recent development of efficient red-shifted calcium probes thus allowing multicolour imaging. ${ }^{5,6}$ To this regard we recently introduced a family of functionalizable red-emitting fluorescent calcium probes named Calcium-Rubies ${ }^{7,} 8$ that were efficiently use in neurobiology ${ }^{9}$ as well as for designing nanosensors. ${ }^{10}$ Even more red-shifted fluorescent probes, especially near-infrared (NIR) emitting ones are highly popular for bio-imaging applications due to their long-wavelength (650-900 nm) excitation and emission, which reduce photo-damage of biological samples, allow deep tissue penetration and avoid background signal from cell auto-fluorescence. ${ }^{11-12}$ In the literature only few examples of NIR emitting fluorescent calcium indicators (Emission $>650 \mathrm{~nm}$ ) are given, among them different fluorophores have been used including squaraine, ${ }^{13}$ cyanine ${ }^{14-15}$ silicon rhodamine ${ }^{16}$ and Bodipy. ${ }^{17}$ Some of these NIR emitting sensors exhibit very good spectral and photophysical properties but none of them are functionalizable. Indeed, functionalization opens new possibilities, for instance, coupling calcium sensors with hydrophilic compounds such as dextrans avoids leakage through cell membranes as well as intracellular compartmentalization and therefore leads to a better diffusion within the neurons. ${ }^{8}$ Moreover, these probes are "turn on" or "turn off" meaning that their emission fluorescence intensity respectively increases or decreases at a given wavelength upon binding with $\mathrm{Ca}^{2+}$. However, ratiometric near-infrared probes that change the intensity ratio at two distinct emission wavelengths upon binding with $\mathrm{Ca}^{2+}$, are missing. ${ }^{18}$ Importantly, the signal of ratiometric probes is independent of many parameters such as probe concentration, photobleaching and some instrumental settings. For these reasons, we herein present Ca-NIR, the first ratiometric fluorescent calcium probe emitting in the near infrared region (Figure 1).
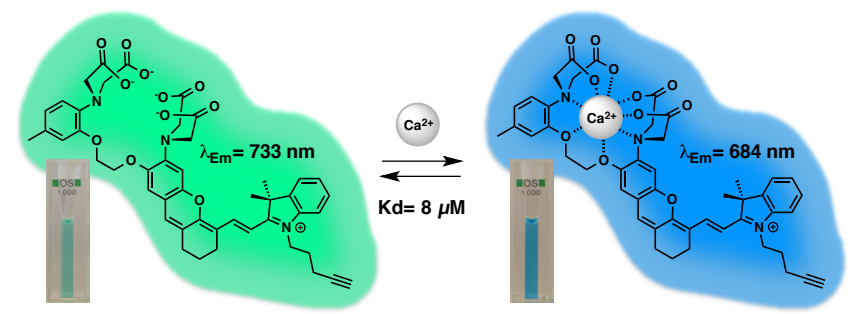

Figure 1. Structure and picture of Ca-NIR solutions $(5 \mu \mathrm{M})$ in absence and in the presence of $\mathrm{Ca}^{2+}$ ions.

Although several efforts have been made to develop new efficient and selective calcium ligands, ${ }^{19,} 20,21$ BAPTA (1,2-bis(o-aminophenoxy)ethane- $N, N, N^{\prime}, N^{\prime}$-tetraacetic acid) remains the basis of efficient fluorescent calcium probes. 


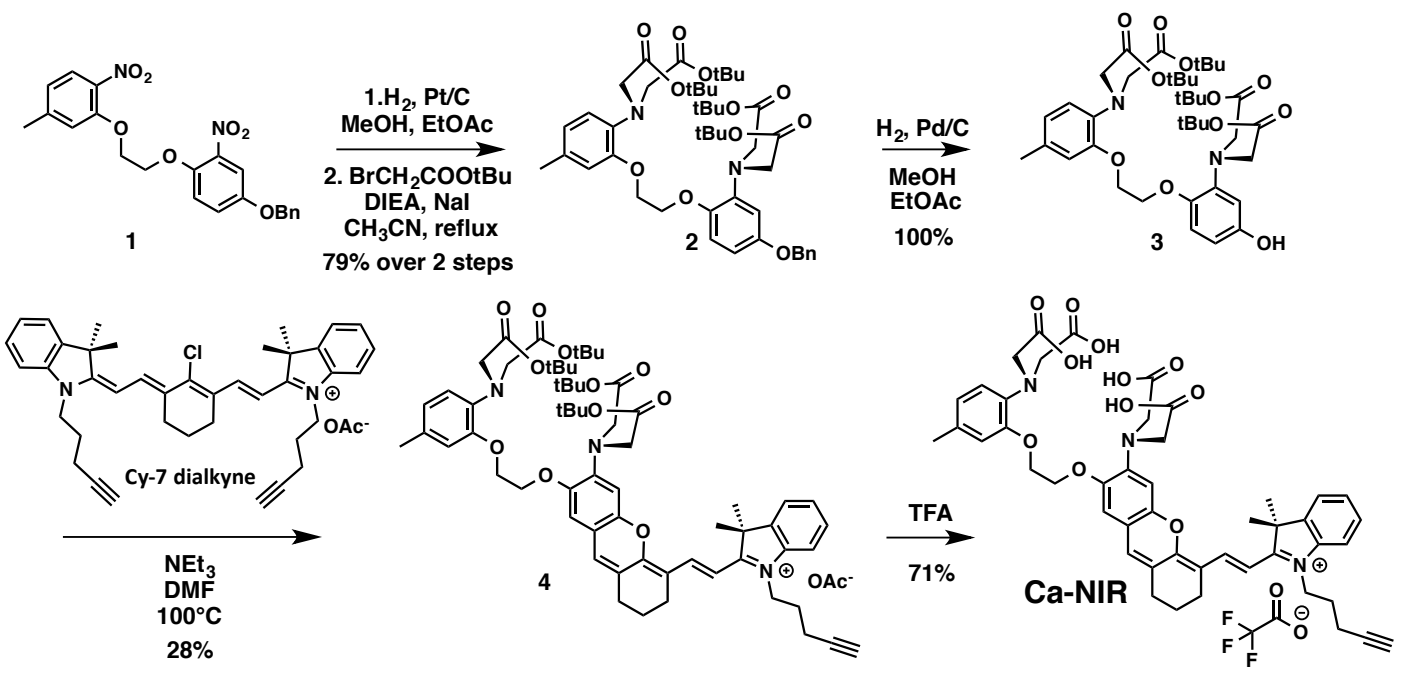

Scheme 1. Synthesis of Ca-NIR

Therefore we aimed at integrating the BAPTA ligand to a NIR emitting fluorophore. In order to obtain a ratiometric probe the fluorophore had to be fused to the BAPTA moiety where a nitrogen and an oxygen of the BAPTA are electron donors, so that when these atoms are involved in the chelation of $\mathrm{Ca}^{2+}$, the whole electronic system of the fluorophore is disturbed and thus change its spectral properties. Dihydroxanthenehemicyanines recently arose as a new class of NIR fluorophores which led to the development of efficient ratiometric probes for in vivo imaging. ${ }^{22}$ Although these sensors are generally based on a hydroxyl electron donor group that is either deprotonated ${ }^{23,24}$ or uncaged, ${ }^{25,26,27}$ introduction of a nitrogen as a donor has also been reported. $^{28,29}$ However, these fluorophores have never been used for designing $\mathrm{Ca}^{2+}$ sensors.

Although new efficient routes have been explored, ${ }^{29}$ these fluorophores are usually obtained by condensation of resorcinol or hydroxyl aniline derivatives on a chloro-cyanine 7 dye in the presence of traces of water. ${ }^{28,30}$ In order to fuse the BAPTA moiety to a dihydroxanthene-hemicyanine fluorophore, a meta-hydroxy-BAPTA had to be obtained. To this end, $\mathbf{1}^{4}$ was involved in a chemoselective reduction of the nitro groups by use of platinum $\left(\mathrm{Pt}^{0}\right)$ as a catalyst. The obtained dianiline was $\mathrm{N}$-alkykated to obtain $\mathbf{2}$. Then the benzyl-protecting group was reduced to provide the desired meta-hydroxy BAPTA 3. The latter was then reacted on a Cy-7 dialkyne in the presence of triethylamine to give 4 . The carboxyl groups were deprotected in the presence of TFA to finally obtain Ca-NIR after purification by reverse-phase chromatography (Scheme 1).

First, Ca-NIR was found to be readily soluble in water (up to 1 $\mathrm{mM}$ ). In calcium free medium, Ca-NIR displays a green jade colour with typical xanthene-shape absorption and emission spectra with maxima at $709 \mathrm{~nm}$ and $733 \mathrm{~nm}$, respectively (Figure $2 \mathrm{~A}$ and $\mathrm{B}$ ). Spectroscopic calcium titration confirmed that the fused structure of BAPTA with dihydroxanthenehemicyanine dye led to a ratiometric probe as upon addition of calcium, Ca-NIR displays an azur colour with a complex absorption spectrum with two peaks at 616 and $666 \mathrm{~nm}$, combined with the appearance of a blue-shifted emission band at $684 \mathrm{~nm}$. Very interestingly, the titration revealed an isobestic point between 630 and $640 \mathrm{~nm}$ that is common wavelength ranges for lasers in bio-imaging. Moreover at these excitation wavelengths, depending on the calcium concentration, Ca-NIR displays two distinct emission bands ( 684 and $733 \mathrm{~nm}$ ) that are well separated ( 50 nm difference) thus offering ideal conditions for ratiometric imaging using conventional microscopy. Titration curves based on decreasing of the $733 \mathrm{~nm}$ band and on increasing of the $684 \mathrm{~nm}$ band fitted with Hill's equations provided dissociation constants of $9.3 \pm 0.5$ and $7.1 \pm 0.2 \mu \mathrm{M}$ respectively which give an average $\mathrm{K}_{\mathrm{D}}$ of $\sim 8 \mu \mathrm{M}$ (Figure $2 \mathrm{C}$ ). Upon protonation in the physiological range ( $\mathrm{pH} 8$ to $\mathrm{pH} 5$ ) absorption and emission spectra are virtually not affected and the pKa was found to be $4.15 \pm 0.08$ (Figure S2 and S3), therefore Ca-NIR is suitable for probing calcium in cells independently from acidification. It is noteworthy that upon protonation the blue-shifted emission band at $684 \mathrm{~nm}$ does not appear and only leads to the decrease of the fluorescence intensity of the red-shifted emission band at $733 \mathrm{~nm}$ (Figure S2). 



Figure 2. Absorption (A) and emission spectra (B) of Ca-NIR $(1 \mu \mathrm{M})$ upon titration with increasing concentration of calcium, 0 (EGTA $1 \mathrm{mM}$ ) to $1 \mathrm{mM}$ $\mathrm{Ca}^{2+}$ (in $30 \mathrm{mM}$ MOPS buffer, $100 \mathrm{mM} \mathrm{KCl}, \mathrm{pH}$ 7.2). (C) Titration curves based on the two emission wavelengths of Ca-NIR ( $1 \mu \mathrm{M})$, and the fits, according to Hill's equation, yielding the Kds. (D) influence of magnesium on the ratiometric calcium sensing of Ca-NIR $(1 \mu \mathrm{M})$. Excitation wavelength was at $600 \mathrm{~nm}$.

According to these results, it can be hypothesized that, in contrast to protonation of nitrogen atom of BAPTA at low $\mathrm{pH}$, chelation of the calcium involves both nitrogen and oxygen atoms, which results in the appearance of the blue-shifted band at $684 \mathrm{~nm}$. Moreover, the presence of well resolved vibrational structure in this absorption band suggests that formation of the Ca-complex leads to a more rigid chromophore. Magnesium $\left(\mathrm{Mg}^{2+}\right)$ is present in cytoplasm of cells and can compete with calcium, for this reason we showed that Ca-NIR was not sensitive to magnesium as the ratio of the two emission bands was not affected in the presence of magnesium (Figure 2D). In order to demonstrate that Ca-NIR is compatible with bio-imaging, it was involved in cellular experiments. Ca-NIR was first incubated in the presence of KB cells (sub-line of the tumour cell line HeLa) for 2 hours and laser scanning confocal microscopy images revealed that despite its limited brightness (see table S1) and its hydrophilic nature due to the presence of several charges, the probe internalized in the cells and display a bright fluorescence in the cytoplasm (See Figure S4). Additionally, the probe displayed a very good photostabilty of both calcium bond and the calcium free forms as continuous scanning with high power laser for 10 min did not affect the intensity of the signals (Figure S6). In a second time, Ca-NIR was added to KB cells in the presence of a calcium free buffer (EGTA) and in the presence of calcium (Figure 3). In order to ensure cytosol delivery of $\mathrm{Ca}^{2+}$ and $\mathrm{Ca}$ NIR probe, digitonin was used, which form transient pores in the cell membranes. In both cases the sensor distributed in the whole cell as the fluorescence signals were detected in the cytoplasm as well as in the nucleus probably due to the permeabilization of the nuclear envelope. Taking advantage of its spectral properties, Ca-NIR was excited at $634 \mathrm{~nm}$ and two emission channels were used (see Figure S5) as the "calcium channel" (A: 650-690 nm) and the "calcium free channel" (B: $700-800 \mathrm{~nm}$ ) thus allowing ratiometric imaging. The results showed that in calcium free conditions, the displayed ratio was low whereas in the presence of calcium much higher ratio values were observed all over the cells.
$650-800 \mathrm{~nm}$

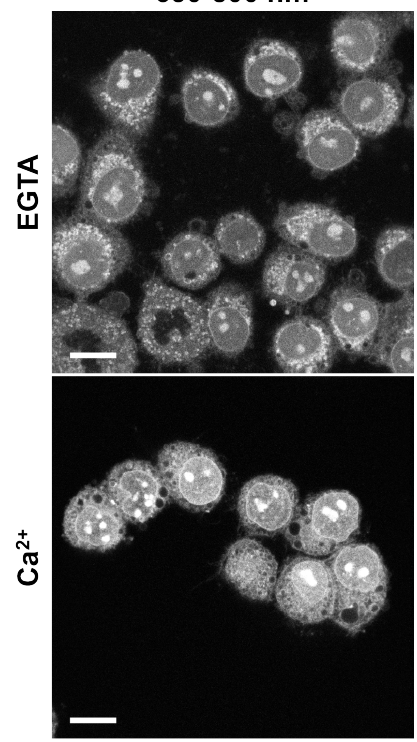

Ratiometric images

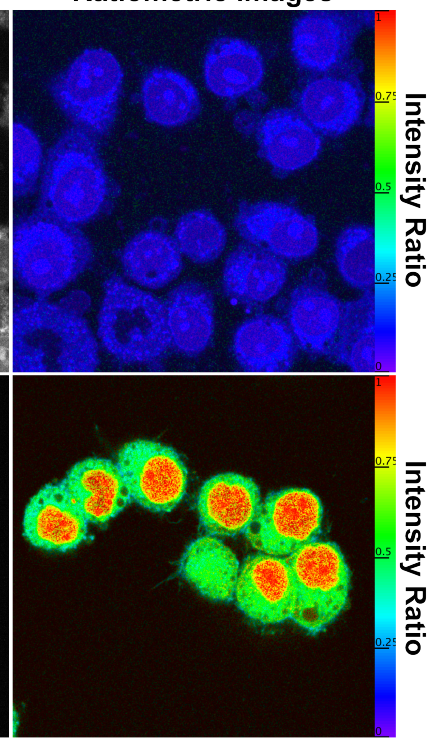

Figure 3. Laser scanning confocal microscopy of $\mathrm{KB}$ cells in the presence of Ca-NIR ( $5 \mu \mathrm{M}$ in MOPS $30 \mathrm{mM}, \mathrm{KCl} 100 \mathrm{mM}, \mathrm{pH} 7.2$, digitonin: $100 \mu \mathrm{g} \cdot \mathrm{mL}^{-1}$ ). Top: EGTA $(1 \mathrm{mM})$ and $\mathrm{Ca}^{2+}(1 \mathrm{mM})$. Left: Sum of channels A $(650-690 \mathrm{~nm})$ and $B(700-800 \mathrm{~nm})$. Right: Ratiometric images obtained by the ratio of channels A/B. Images were taken $10 \mathrm{~min}$ after addition of Ca-NIR. Scale bar is $15 \mu \mathrm{m}$.

The heterogeneity in the emission colour between the cytoplasm and nucleus could reflect either differences in $\mathrm{Ca}^{2+}$ concentration or the presence of lipid membrane structures e.g. endoplasmic reticulum etc, inside the cytoplasm that could slightly inhibit response of the probe to $\mathrm{Ca}^{2+}$. Co-staining of cells with Hoechst as a nuclear marker revealed that Ca-NIR preferentially localized in the nuclear envelope as well as in the nucleoli (Figure S8). These compartments display a high ratio colour in ratiometric imaging as they are known to be potent reservoirs of calcium. ${ }^{31,32}$ Additionally we investigated on the influence of DNA on Ca-NIR and showed that even in the presence of a large excess of DNA the sensing ability of CaNIR was not affected (Figure S9). These results tend to show that Ca-NIR is able to map the distribution of calcium concentration within the cells using the $630-640 \mathrm{~nm}$ lasers and the NIR region of the spectrum, therefore allowing the use a wide range of other colours for multicolour imaging. In conclusion, Ca-NIR is the first ratiometric calcium probe emitting in the near infrared; its Kd ranges from 7 to $9 \mu \mathrm{M}$ and is compatible with cellular ratiometric imaging. Additionally, its alkyne side-arm leaves the possibility for further functionalization with dextran or sub-cellular targeting molecules. As far as our knowledge, Ca-NIR is the first nearinfrared ratiometric fluorescent calcium probe. 


\section{Acknowledgment}

This work was supported by ERC Consolidator grant BrightSens 648528. We thank Dr. Christian Wilms for his suggestions, Pichandi Ashokkumar for the cell culture, Romain Vauchelles for his assistance at the PIQ platform and for the Ratio Intensity Color Version 2.5 plugin, Sabine Lang for the mass measurements and Bohdan Wasylyk for providing the KB cells.

\section{Notes and references}

1 D. E. Clapham, Cell, 2007, 131, 1047-1058.

2 G. R. C. Hamilton, S. K. Sahoo, S. Kamila, N. Singh, N. Kaur, B. W. Hyland and J. F. Callan, Chem. Soc. Rev., 2015, 44, 4415-4432.

3 J. Yin, Y. Hu and J. Yoon, Chem. Soc. Rev., 2015, 44, 4619-4644.

4 G. Grynkiewicz, M. Poenie and R. Y. Tsien, J. Biol. Chem., 1985, 260, 3440-3450.

5 M. Oheim, M. van 't Hoff, A. Feltz, A. Zamaleeva, J.-M. Mallet and M. Collot, Biochim. Biophys. Acta-Mol. Cell Res., 2014, 843, 2284-2306.

6 K. Hirabayashi, K. Hanaoka, T. Egawa, C. Kobayashi, S. Takahashi, T. Komatsu, T. Ueno, T. Terai, Y. Ikegaya, T. Nagano and Y. Urano, Cell Calcium, 2016, 60, 256-265.

7 M. Collot, C. Loukou, A. V. Yakovlev, C. D. Wilms, D. Li, A. Evrard, A. Zamaleeva, L. Bourdieu, J.-F. Leger, N. Ropert, J. Eilers, M. Oheim, A. Feltz and J.-M. Mallet, J. Am. Chem. Soc., 2012, 134, 14923-14931.

8 M. Collot, C. D. Wilms, A. Bentkhayet, P. Marcaggi, K. Couchman, S. Charpak, S. Dieudonné, M. Häusser, A. Feltz and J.-M. Mallet, eLife, 2015, 4, e05808.

9 Y. Otsu, K. Couchman, D. G. Lyons, M. Collot, A. Agarwal, J.-M. Mallet, F. W. Pfrieger, D. E. Bergles and S. Charpak, Nat. Neurosci., 2015, 18, 210-218.

10 A. I. Zamaleeva, M. Collot, E. Bahembera, C. Tisseyre, P. Rostaing, A. V. Yakovlev, M. Oheim, M. de Waard, J.-M. Mallet and A. Feltz, Nano Lett., 2014, 14, 2994-3001.

11 L. Yuan, W. Lin, K. Zheng, L. He and W. Huang, Chem. Soc. Rev., 2012, 42, 622-661.

12 K. Umezawa, D. Citterio and K. Suzuki, Anal. Sci., 2014, 30, 327349.

13 E. U. Akkaya and S. Turkyilmaz, Tetrahedron Lett., 1997, 38, 4513-4516.

14 B. Ozmen and E. U. Akkaya, Tetrahedron Lett., 2000, 41, 91859188.

15 A. L. Ellis, J. Christian Mason, H.-W. Lee, L. Strekowski, G. Patonay, H. Choi and J. J. Yang, Talanta, 2002, 56, 1099-1107.

16 T. Egawa, K. Hanaoka, Y. Koide, S. Ujita, N. Takahashi, Y. Ikegaya, N. Matsuki, T. Terai, T. Ueno, T. Komatsu and T. Nagano, J. Am. Chem. Soc., 2011, 133, 14157-14159.

17 A. Matsui, K. Umezawa, Y. Shindo, T. Fujii, D. Citterio, K. Oka and K. Suzuki, Chem. Commun., 2011, 47, 10407-10409.

18 Summary of Molecular Probes fluorescent $\mathrm{Ca} 2+$ indicatorsTable 19.1 https://www.thermofisher.com/fr/fr/home/references/molecul ar-probes-the-handbook/tables/summary-of-fluorescent-ca2indicators-available-from-molecular-probes.html, (accessed May 11, 2017).

19 M. Collot, C. Wilms and J.-M. Mallet, RSC Adv., 2014, 5, 69937000.

20 E. Arunkumar, A. Ajayaghosh and J. Daub, J. Am. Chem. Soc., 2005, 127, 3156-3164.
21 B. Sui, X. Liu, M. Wang and K. D. Belfield, Chem. - Eur. J., 2016, 22, 10351-10354

22 L. Yuan, W. Lin, S. Zhao, W. Gao, B. Chen, L. He and S. Zhu, J. Am. Chem. Soc., 2012, 134, 13510-13523.

23 Y. Li, Y. Wang, S. Yang, Y. Zhao, L. Yuan, J. Zheng and R. Yang, Anal. Chem., 2015, 87, 2495-2503.

24 Q. Wan, S. Chen, W. Shi, L. Li and H. Ma, Angew. Chem. Int. Ed., 2014, 53, 10916-10920.

25 L. Zhang, X. E. Zheng, F. Zou, Y. Shang, W. Meng, E. Lai, Z. Xu, Y. Liu and J. Zhao, Sci. Rep., 2016, 6, 18868.

26 Z. Li, X. He, Z. Wang, R. Yang, W. Shi and H. Ma, Biosens. Bioelectron., 2015, 63, 112-116.

27 C. Han, H. Yang, M. Chen, Q. Su, W. Feng and F. Li, ACS Appl. Mater. Interfaces, 2015, 7, 27968-27975.

28 H. Chen, W. Lin, H. Cui and W. Jiang, Chem. - Eur. J., 2015, 21 , 733-745.

29 M. J. H. Ong, R. Srinivasan, A. Romieu and J.-A. Richard, Org. Lett., 2016, 18, 5122-5125.

30 J.-A. Richard, Org. Biomol. Chem., 2015, 13, 8169-8172.

31 J.-P. Mauger, Biol. Cell, 2012, 104, 70-83.

32 D. M. O’Malley, J. Neurosci., 1994, 14, 5741-5758. 